International Journal of Methods in Psychiatric Research

Int. J. Methods Psychiatr. Res. 15(4): 207-214 (2006)

Published online in Wiley InterScience

(www.interscience.wiley.com) DOI: 10.1002/mpr.198

\title{
The Computerized MacArthur Story
}

\section{Stem Battery - a pilot study of a novel}

\section{medium for assessing children's representations of relationships}

\author{
HELEN MINNIS, ${ }_{1}$ REUBEN MILLWARD, ${ }^{2}$ CLAIRE SINCLAIR, ${ }^{3}$ EILIS KENNEDY, ${ }^{4}$ ANNE GREIG, ${ }^{5}$ \\ KATE TOWLSON, ${ }^{2}$ WARREN READ, ${ }^{6}$ JONATHAN HILL ${ }^{7}$ \\ 1 University of Glasgow Section of Psychological Medicine, UK \\ 2 Lomond and Argyll Primary Care Trust \\ 3 NHS Lanarkshire, Motherwell \\ 4 Tavistock Clinic, London \\ 5 Argyll and Bute Council, Helensburgh \\ 6 ClarksonRead Consulting, Glasgow \\ 7 University of Manchester, Manchester
}

\begin{abstract}
Story stem measures allow the assessment of children's representations of relationship functioning, but are expensive and time-consuming to administer. We developed a computerized story stem measure which does not require specific training for administrators and which allows the child to produce their own animated, narrated story completion. This paper describes, firstly, the reliability of the Computerized MacArthur Story Stem Battery (CMSSB) and, secondly, a preliminary comparison of children in foster care and school controls on narrative coherence, intentionality and avoidance. The CMSSB showed good inter-rater reliability. A group of children in foster care showed significantly poorer coherence of narrative, less intentionality and greater avoidance on the CMSSB compared to a school comparison group. Copyright (C) 2006 John Wiley $\mathcal{B}$ Sons, Ltd.
\end{abstract}

Key words: foster care, attachment, story stems

The measurement of attachment has classically been made by observation of infant behaviour in relation to a parent or caregiver (Ainsworth, 1979b) but measurement becomes more complex after infancy as the behavioural indices of proximity-seeking and contact maintenance displayed by the younger child become less apparent (Solomon and George, 1999). However, young children are thought to encode knowledge about their relationships with attachment figures through symbolic forms of representation (Bretherton, 1985) and various measures of attachment in middle childhood have been based on this theoretical model. These representational measures, which have included pictureresponse procedures (Jacobsen et al., 1994; Main et al., 1985; Solomon and George, 1999) and doll-play measures (Bretherton, 1985; Solomon and George, 1999), have demonstrated reasonable reliability, stability over time and validity against behavioural measures (Solomon and George, 1999). For example, Goldwyn et al. (2000) reported associations between disorganized attachment rated from the Manchester Child Attachment Story Task in 5-7 year olds and unresolved status in mothers assessed using the Adult Attachment Interview. 


\section{The MacArthur Story Stem Battery (MSSB)}

The MacArthur Story Stem Battery is usually used with children aged 4 to 8 and uses doll play to assess children's representations of relationships. It aims to evaluate not only attachment-related indices, but also aspects of moral, emotional and social development including affect expression, affect regulation, themes of aggression and conflict and the involvement of parents. It has been used widely in both clinical work and research, including studies of the internal representations of children from normative samples (Woolgar et al., 2001), maltreated children (Macfie et al., 1999), children exposed to parental conflict (Grych et al., 2002) and children with disruptive behavioural disorders (Warren et al., 1996; Von Klitzing et al., 2000). It has been shown to predict behaviour problems (Von Klitzing et al., 2000; Warren et al., 1996) and anxiety in children (Warren et al., 2000).

The administration process involves telling a child the scripted stem of a story, using simple dolls as props. The child is then asked to 'show and tell' the psychiatrist 'what happens next'. The child's completion of each scenario is recorded on video and analysed later by a trained evaluator using a scoring template. After a warm-up scenario, which is not rated, usually eight to 12 story stems are used for rating. Stems depict a range of moral and relationship dilemmas. For example, in a stem titled Lost Keys, the administrator describes Mum and Dad in the kitchen, using dolls. The script goes as follows:

Mum: 'You've lost my keys!'

Dad: 'I have not!'

Mum: 'Yes you have, you always lose my keys!'

The administrator then hands the child the dolls saying 'show me and tell me what happens next.'

At predetermined points in the child's narrative, the administrator interjects with scripted prompts. For example, at the end of the Lost Keys scenario, when the child appears to have finished the story, the administrator asks 'What happened to the keys?'

In common with other doll-play measures, there are various difficulties with administering and rating the MSSB: differences in administration of stems can affect interpretation of children's responses and the package is expensive to employ, requiring approximately one hour per child from a trained administrator. The use of doll-play techniques in large epidemiological studies is therefore rare, though not unknown (Von Klitzing et al., 2000). Because representational measures are a rich source of information about children's relationships (Solomon and George, 1999), it seems a worthwhile endeavour to continue to try to develop such measures using new media.

\section{Computers in the psychological assessment} of children

Children enjoy interacting with computers as evidenced by the growth in computer games and educational software packages. Computers are increasingly being used in psychiatric assessment with children and young people. For example, the Voice-DISC - a measure of DSM-IV diagnoses in which respondents selfadminister in interaction with a voice - has been shown to be highly acceptable and appears to be at least as reliable as the interview version. Its ability to be administered to large groups of children in a school setting made it possible to generate psychiatric diagnoses within a large epidemiological study (West et al., 2003). A computerized version of the MSSB could potentially allow the testing of larger groups of children under the supervision of one less highly trained administrator, hence with lower cost. Secondly, it would allow the carefully designed MSSB scripts to be delivered entirely consistently. Thirdly, our previous work with computer-based questionnaire design (Truman et al., 2003) suggests that the child may feel more at ease and be less focussed on being in a 'test' situation.

We developed a computerized version based on the doll-play MSSB (the CMSSB) in an attempt to reduce the amount of therapist's time needed for administration, eliminate the need for specific training of administrators and potentially increase the child's motivation to participate.

\section{The Computerized MacArthur Story Stem Battery (CMSSB)}

A prototype CMSSB was piloted for acceptability with 10 volunteer children who were not attending child and adolescent psychiatry clinics. After discussion with members of the MacArthur group, we decided only to animate six of the story stems as these seem to be emerging in current research as the most replicable and reliable. These stems are: The Lost Keys, The Burnt Hand, The Scary Dog, The Sweet Shop, Fight with a Friend and Mum's Headache. 
The CMSSB has two warm-up sections: one in which the administrator shows the child how to move the characters on the screen until the child is comfortable with the technology and a second (The Birthday Party) in which the child completes a story stem that does not contain a relationship dilemma and is not rated. These introductory sections take about 10 minutes. From then on, the child essentially interacts only with the computer, although in our study there was always an administrator on hand in case of difficulty. The child is shown an animated beginning of a story using simple drawings on the computer screen (Figure 1). A voice-over asks the child to 'show me and tell me what happens next'.

It was a challenge to mimic the prompts provided in the doll-play original, as a computer cannot respond to an infinite number of possible child stories, therefore a simply animated character called 'Mr. Query' (Figure 2) was developed to provide structured prompts. $\mathrm{Mr}$ Query, who is probably most akin to the 'helpers' found in common word-processing software, is introduced during a warm-up scenario as someone who will be popping in and out and won't see the action so might need to ask questions now and again. Mr Query pops up after a specifically timed period and asks whether the child is finished telling the story. The child has the option of clicking that 'yes' s/he is finished, 'no' s/he is not finished or of ignoring Mr Query completely. If the story has a scripted prompt, Mr Query then introduces

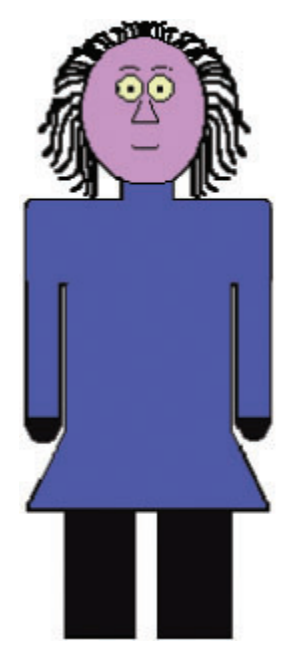

Figure 1. Example of simple line drawings used in CMSSB. the prompt if the child has indicated that s/he has finished telling the story, or alternatively after a further short period of time has elapsed. Various time periods were tried during the pilot of the prototype until we achieved the most acceptable structure for the participants where Mr Query's interjections supported the child's interaction but did not appear to disrupt the flow of narrative.

The child's vocal responses and the way s/he moves the characters on the screen, using the mouse, are recorded simultaneously and downloaded onto $\mathrm{CD}$. When raters of the CMSSB open the child's file on CD, they see an animated story completion on the screen, narrated by the child. Rating can then be carried out as for the doll-play version.

Asking children to respond to predetermined prompts on a computer screen is clearly very different from engaging with them in play. We therefore conducted a study to find out whether children provide responses using the CMSSB that can be rated using an established MSSB scoring system, and whether vulnerable children (in foster care) provide narratives that differ in predicted ways from low risk children (in schools).

\section{The high-risk sample}

Children in foster care are clearly a vulnerable group because the majority have a history of abuse and neglect (Minnis et al., 2001) and they have high rates of mental health problems (Meltzer et al., 2003). The effects of disturbances in the early social relationships of children in foster care are difficult to measure using standard psychiatric tools and measures of attachment may be particularly relevant in this group (Rushton and Minnis, 2002). Little is known about the attachment

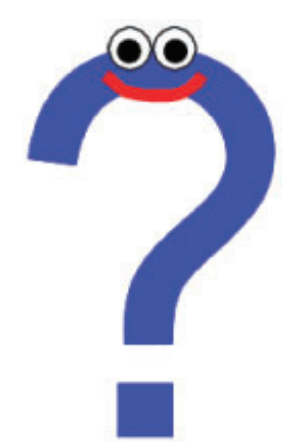

Figure 2. Mr Query. 
patterns, in middle childhood, of children in foster care although the study of attachments of fostered children in infancy has demonstrated concordance between infant attachment and caregiver state of mind with respect to attachment (Dozier et al., 2001). Late-placed adopted children, many of whom have experienced multiple foster placements prior to their adoption, are more likely to demonstrate avoidance of dilemmas presented in story stems compared to infant adoptees (Hodges et al., 2003). Children in foster care have almost universally come from family backgrounds in which parent-infant interaction was deemed grossly inadequate and therefore are likely to have little experience of sensitive parenting in early life. We hypothesized, therefore, that children in foster care would demonstrate poorer ratings on attachment-related indices compared to school controls.

In the comparison of the fostered children and the school control group the focus was on measures of avoidance, coherence and intentionality. For simplicity, in this pilot study, we did not attempt to analyse the children's responses to individual stories, but examined scores across all stems. There are strong theoretical reasons why children in foster care would be expected to rate highly on avoidance. Children in foster care have almost universally experienced abuse and/or neglect (Minnis et al., 2001) and many have experienced rejection to a degree that has resulted in their ejection or removal from the family. The experience of rejection is associated with placement breakdown in adoption, especially if children have been singled out for rejection over siblings who have remained in the birth family (Dance et al., 2002). The mechanisms for this association between early rejection and later placement breakdown are unknown but avoidant attachment patterns may have a role to play. Avoidant attachment in mother-infant dyads has been shown to be associated with maternal rejection (Ainsworth, 1979a) and maltreated children's narratives contain more avoidance than school comparisons (Hodges and Steele, 2000). Conversely, secure maternal attachment status during pregnancy predicted acknowledgement of distress (low avoidance) in responses to story challenges at age 11 in a low-risk sample (Steele et al., 2002).

Coherence is a central concept in the assessment of adult attachment (Main et al., 1985; Main and Goldwyn, 1984). The key is the provision of an account that is relevant, which can be readily followed, and is not cut short by avoidance of the topic nor derailed by preoc- cupation in the moment. Applied to children's responses to MacArthur Story Stems, it refers to their ability to acknowledge the implications of the dilemma presented, and to provide a relevant and adequate development of the narrative, and bring it to a conclusion. Steele et al (Steele et al., 2002) reported that secure maternal attachment antentally predicted 11-year-old children's ability to elaborate a resolution when challenged to respond to moderately distressing cartoon sequences.

Intentionality refers to the extent to which the child provides an account that is portrayed or told in terms of the participants' motives, emotions or beliefs. It provides an index of mentalizing, which may be promoted within secure attachment relationships (Fonagy and Target, 1997). Hill et al. (2000) found that secure attachment in infancy predicted higher intentionality in five year olds' responses to a request to show what happens in the family when there is a 'bad and nasty time'.

Children in foster care have almost universally come from family backgrounds in which parent-infant interaction was deemed grossly inadequate or dangerous and children in the foster care sample are likely to have little experience of sensitive parenting in the birth family. We hypothesized, therefore, that story completion by children in foster care would demonstrate differences on attachment-related indices compared to school controls.

The aims of the study were twofold: firstly we predicted that, because of the entirely consistent administration of the CMSSB, inter-rater reliability would be good. Secondly, in order to examine the criterion validity of the CMSSB, we wished to test the preliminary hypothesis that it would detect poor coherence, intentionality and avoidance of relationship dilemmas in the stories of fostered children compared to children from the general population.

\section{Methods}

Sample

The study involved 34 children aged four to nine years: 17 children in foster care and 17 control children from a local primary school, selected for its high level of deprivation, whose parents volunteered their children to participate (see Table 1). Fostered children were approached through a Scottish Social Work Department and $63 \%$ of their parents, or social worker if in loco parentis, gave consent for their participation. The 
study had the ethical approval of the local medical and educational ethics committees.

\section{Measures}

The British Picture Vocabulary Scale (BPVS) is a wellvalidated test of verbal comprehension (Atkinson, 1992). Children were asked to complete this prior to completing the CMSSB.

The CMSSB: six story stems were administered to the children on a computer screen as described earlier. The responses were rated using a coding scheme designed to capture a range of indices of the quality of the narrative provided by the child (University of Liverpool, 2000). All of the raters were trained by JH and ratings were made using a detailed manual. Only the scales used to compare the fostered children and their controls are described in detail here. Ratings for each of avoidance, coherence and intentionality scales are made on 12-point scales.

High avoidance is rated where a problem is removed rather than confronted. The problem may be the one presented in the stem, or generated by the child within the story. Generally the avoidant manoeuvre alters the facts and the emotional implications of the facts, so that for example, the response to the child in Scary Dog saying 'oh I'm so scared' is to say that the dog wags its tail and the child pats it. Ratings in the range ' $1-3$ ' are made where there is considerable emotional expression and the implications of the challenge in the story stem are fully taken up. A rating in the '4-6' range reflects a restricted but not clearly avoidant story. A rating between '7-9' indicates definite avoidance, but it does not dominate, whereas ratings in the '10-12' range indicate strong and dominant avoidance.

The intentionality scale reflects the extent to which the characters in the story are portrayed by the child as having states of mind and intentions. Contributions to ratings in the highest range (10-12) are made by explicit references to the feelings, thoughts or motives of the characters. For example the child comments ' $\mathrm{He}$ runs to his mother because he is frightened.' Ratings in the 7-9 range are made when intentionality can be readily referred from the context of the actions, the emotions expressed, or what is said. For instance one character says to another, 'Don't you dare talk to me like that!' and attacks him. It is clear that the state of mind has to do with humiliation or feeling insulted although that is not stated. In the 4-6 range actions appear to be determined to a greater or lesser extent by physical circumstances rather than by the states of mind of the participants, but the symbolic function of the characters and props is preserved. Here typically the child describes characters along the lines of 'and then he sat down' because there is a chair available but without relevance to point reached in the story. In the lowest range there are actions without any obvious intentionality, and it is not clear that the doll figures and props are being used symbolically. For example the child puts a doll in the oven, or on the roof of the house.

The coherence scale assesses the quality of the narrative in terms of the logic of the sequences, the effectiveness of affect regulation, and the appropriateness of the portrayed actions. Ratings in the highest quadrant are made for an elaborated story in which some story lines are developed, there is clear portrayal of emotions that are regulated and dilemmas are resolved effectively within the story line. Ratings in the 7-9 quadrant reflect a story that is relatively brief and unelaborated but in which the logic of the sequences is clear, and any dilemmas are resolved. A rating in the 4-6 range is made where there are definitely elements that reduce coherence, without undermining the whole story. For example there may be a phase in which important elements of a dilemma are bypassed, or where there is a marked and inappropriate escalation of aggression, but other phases where the links are clear and appropriate. Ratings in the 1-3 range reflect persistent and/or severe disruption of the logic of the account, for example by unaccounted shifts of theme, sudden escalations of negative affect, or events that apparently have no cause. This scale tends to be associated with verbal IQ, therefore the BPVS was included as a potential confounder in regression analyses.

After completing the CMSSB, children were asked two simple questions: 'did you enjoy doing the test?' and 'did you find it hard?'

Stories were transcribed and rated by RM, CS, AG and EK for a range of indices including coherence of narrative, intentionality and avoidance of the dilemma presented in the story. Raters were blind to the care status of the child. A random sample of $10 \%$ of stories, selected from all participants, was allocated to a second rater who was blind to the first rating.

\section{Statistical analysis - the reliability study}

For analysis, the scores for individual stories on continuous scales were summed to form composite scales 
- for example, the coherence score for the Scary Dog story has a possible range of 1 to 12 , and the composite coherence score for all six scales has a possible range of 1 to 72 . Interrater reliability was assessed using the limits of agreement method. This method avoids the systematic biases possible in correlational methods of assessing intrarater reliability by relying on mean differences in scores between raters but also assesses the closeness of ratings by placing a 95\% confidence interval around the mean difference (Bland and Altman, 1986). The sample size was the same as that in Bland and Altman's original study.

\section{Statistical analysis - the between-group comparison study}

Between-group comparisons used t-tests and, adjusting for potential confounders (age, gender and BPVS score), multiple regression analysis. Our only between-group comparisons were on the coherence, intentionality and avoidance scales, so no adjustment was necessary for multiple testing. All analyses were carried out in SPSS (version 11).

On examination of the distributions of the mean avoidance, coherence and intentionality scores there were no outliers nor were there indications of skewness. There were strong associations of each of the scales with age and with BPVS but not with gender. Analyses were conducted using analysis of covariance with foster care versus control as the grouping variable, and age of the child and verbal ability as covariates.

\section{Results}

All participants found the measure acceptable and easy to use. The samples were similar in age, gender and verbal comprehension (see Table 1).

\section{Reliability study}

Agreement between raters was good on all subscales (see Table 2): the mean difference between raters did not exceed $0.02 \%$ of the total scale range.

Table 1. Group comparisons for fostered $(n=17)$ versus school children $(n=17)$

\begin{tabular}{lccr}
\hline Variable & $\begin{array}{l}\text { School children; mean } \\
(\mathrm{sd}) \text { or percentage }\end{array}$ & $\begin{array}{l}\text { Fostered children; mean } \\
(\mathrm{sd}) \text { or percentage }\end{array}$ & $\mathrm{p}$ \\
\hline Age & $8.94(2.66)$ & $7.47(2.09)$ & 0.061 \\
Gender (percentage boys) & $40.0 \%$ & $62.5 \%$ & 0.029 \\
Verbal comprehension (BPVS score) & $7.40(3.26)$ & $7.41(2.55)$ & 0.987 \\
\hline
\end{tabular}

Table 2. Interrater reliability using limits of agreement method

\begin{tabular}{lccc}
\hline Rating scale & $\begin{array}{l}\text { Mean difference between } \\
\text { ratings (95\% confidence } \\
\text { interval) }\end{array}$ & $\begin{array}{l}\text { Range of composite scale } \\
\text { (sum of six stories) }\end{array}$ & $\begin{array}{l}\text { Mean difference (percentage } \\
\text { of total scale range) }\end{array}$ \\
\hline Coherence & $0.73(-5.62-1.52)$ & $1-72$ & 0.01 \\
Denial/avoidance & $1.13(0.0035-2.22)$ & $1-72$ & 0.016 \\
Intentionality of action & $0.19(-0.8-1.22)$ & $1-72$ & 0.003
\end{tabular}

Table 3. Group means for avoidance, coherence and intentionality

\begin{tabular}{lcccr}
\hline Scale & \multicolumn{2}{c}{$\begin{array}{l}\text { School children; (mean, sd) } \\
\mathrm{N}=15\end{array}$} & $\begin{array}{l}\text { Fostered children; (mean, sd) } \\
\mathrm{N}=16\end{array}$ & $\begin{array}{l}\text { Adjusted difference* } \\
\text { (95\% CI) }\end{array}$ \\
\hline Mean avoidance & $6.44(2.85)$ & $8.60(3.14)$ & $-2.50(-4.68--0.33)$ & 0.025 \\
Mean coherence & $6.33(2.53)$ & $4.42(2.61)$ & $1.89(0.20-3.57)$ & 0.029 \\
Mean intentionality & $6.13(2.23)$ & $4.60(2.27)$ & $1.87(0.32-3.42)$ & 0.020 \\
\hline
\end{tabular}

* Adjusted for age and BPVS score. 
Between-group comparisons

Fostered children's CMSSB narratives demonstrated less intentionality, more avoidance and were significantly less coherent compared to control children (Table 3).

\section{Discussion}

The CMSSB appears to deliver an assessment of children's representations of relationships using a new medium - a computer software package. All participants were willing and able to use the technology without difficulty and appeared to find it both easy to use and enjoyable. Although approximately 10 minutes with an administrator is required to introduce the child to the package, the CMSSB does not require specific training for administrators other than basic orientation towards the software. Theoretically, more than one child could work on the package with one administrator as long as the introductory periods were staggered.

Interrater reliability was good for all indices and excellent for certain ratings. It is tempting to speculate that rating might be more reliable for the CMSSB compared to the original doll-play version because of the standardized administration but future research comparing the measures will be required to determine whether this is the case. Such research - and research in which the CMSSB is tested against other measures of attachment and relationship functioning - will be essential in deciding whether the CMSSB is actually testing the same domains as the doll-play original, in other words, whether this is simply a new medium for an established measure or whether it should be seen as a different instrument. The ability of the CMSSB to distinguish between a high-risk and general population sample on a hypothesis driven between group comparison suggests that the former may be the case, but further research comparing the CMSSB with the MSSB will be required.

The results indicate that fostered children are performing less well on this test of narrative coherence, intentionality and avoidance compared to their school peers, controlling for age and verbal comprehension. The finding that fostered children demonstrate greater avoidance of relationship dilemmas in CMSSB stories is unsurprising when their likely histories of early abuse, neglect or rejection are taken into account. Narrative coherence has been shown to be highly correlated with attachment patterns in adults (Main et al., 1985) but the case for this has not been so clearly made, as yet, in children despite suggestions of this in recent literature (Green et al., 2000). Recent evidence suggests that intentionality - apparently an index of mentalizing ability - is associated with security of attachment (Hill et al. 2006, submitted to Child Development) in children. It seems logical that children in foster care, who are likely to have a poorer early relationship history, would perform less well on measures of attachment. Such children, however, may have missed out on crucial aspects of parent-infant interaction which have resulted in less adequate general verbal skills (Trevarthen and Aitken, 2001) not fully controlled for in the BPVS. Whether or not these findings are direct measures of the attachment system will need to be tested in future research.

Despite these limitations, our preliminary results suggest that the CMSSB may be a reliable and potentially cost-effective tool for gaining insight into children's relationship functioning and has the potential to be used in a range of settings in which looked after children are assessed such as CAMHS and community paediatric clinics.

\section{Acknowledgements}

The authors are grateful to the Mental Health Development Fund (Scottish Executive), who provided funding, the participants, the staff of West Dunbartonshire Social Work Department, Education Department and participating schools and David Young for statistical advice.

The roles of the contributors were as follows: HM initiated core ideas, analysed the data, participated in developing the protocol, participated in writing the paper and supervised RM, CS, EK and AG in rating the CMSSB. HM is guarantor. RM, EK and CS discussed core ideas, participated in developing the protocol, administered data collection, rated the CMSSB and participated in writing the paper. AG discussed core ideas, rated the CMSSB and participated in writing the paper. WR discussed core ideas, developed the CMSSB and participated in writing the paper. $\mathrm{KT}$ and $\mathrm{JH}$ discussed core ideas, participated in developing the protocol and in writing the paper. The coding system was devised by JH and staff of the Child and Family Center, Menninger Clinic, Topeka, Kansas, USA.

\section{References}

Ainsworth MD. Infant-mother attachment. American Psychologist 1979; 34(10): 932-7.

Atkinson L. The British Picture Vocabulary Scale. Brit J Disord Comm 1992; 26(3): 369-72.

Bland JM, Altman DG. Statistical methods for assessing agreement between two methods of clinical measurement. Lancet 1986; 1(8476): 307-10. 
Bretherton I. Attachment theory: retrospect and prospect. In I Bretherton, E Waters (eds) Monographs of the Society for Research in Child Development, 50: 1-2, Serial No 209, 1985, pp. 3-35.

Dance C, Rushton A, Quinton D. Emotional abuse in early childhood: relationships with progress in subsequent family placement. J Child Psychol Psychiatr 2002; 43(3): 395-407.

Dozier M, Stovall KC, Albus KE, Bates B. Attachment for infants in foster care: the role of caregiver state of mind. Child Dev 2001; 72(5): 1467-77.

Fonagy P, Target M. Attachment and reflection function: their role in self-organization. Dev Psychopathol 1997; 9: 679-700.

Goldwyn R, Stanley C, Smith V, Green J. The Manchester Child Attachment Story Task: relationship with parental AAI, SAT and child behaviour. Attachment and Hum Dev, 2000; 2(1): 71-84.

Green J, Stanley C, Smith V, Goldwyn R. A new method of evaluating attachment representations in the young school age children: The Manchester Child Attachment Story Task (MCAST). Attachment and Hum Dev 2000; 2: $48-70$.

Grych JH, Wachsmuth-Schlaefer T, Klockow LL. Interparental aggression and young children's respresentations of family relationships. J Family Psycholy 2002; 16(3): 259-72.

Hill J, Hoover D, Taliaferro G. Revised Manual for the MacArthur Narrative Completion Task. Menninger Clinic Technical Report. Report \#00-0035, Menninger Clinic Houston, 2000.

Hill J, Murray L, Leidecker V, Sharp H. Infant attachment, mentalisation and resilience at age 5. Paper presented at the $17^{\text {th }}$ International Association for child and Adolescent Psychiatry and Allied Professions Congress. Melbourne, Australia.

Jacobsen T, Edelstein W, Hofmann V. A longitudinal representations of attachment in childhood and cognitive functioning in childhood and adolescence. Dev Psychol 1994; 30: 112-24.

Macfie J, Toth SL, Rogosh F. Effect of maltreatment of preschoolers' narrative representations of responses to relieve distress and of role reversal. Dev Psychol, 1999; 35(2): 460-5.

Main M, Goldwyn R. Predicting rejection of her infant from mother's respresentation of her own experience: implications for the abused-abusing intergenerational cycle. Child Abuse and Neglect 1984; 8(2): 203-17.

Main M, Kaplan N, Cassidy J. Security in infancy, childhood and adulthood: a move to the level of representation. In I Bretherton, E Waters (eds) Monographs of the Society for Research in Child Development 50[1-2]: 66-104, 1985.
Meltzer H, Gatward R, Corbin T, Goodman R, Ford T. The Mental Health of Young People Looked After by Local Authorities in England. London: Office For National Statistics, 2003.

Minnis H, Pelosi A, Knapp M, Dunn J. Mental health and foster carer training. Arch of Dis Childhood 2001; 84: 302-6.

Rushton A, Minnis H. Residential and Foster Family Care. In M Rutter, E Taylor, L Hersov (eds) Child and Adolescent Psychiatry: Blackwell, London Modern Approaches. 4 edn. London: 2002.

Solomon J, George C. The measure of attachment security in infancy and childhood. In J Cassidy, PR Shaver (eds) Handbook of Attachment. New York: Guilford Press, 1999, pp. 287-316.

Steele M, Steele H, Johansson M. Maternal predictors of children's social cognition: an attachment perspective. J Child Psychol Psychiatr 2002; 43(7): 861-72.

Trevarthen C, Aitken K. Infant intersubjectivity: research, theory, and clinical applications. J Child Psychol Psychiatr 2001; 42(1): 3-48.

Truman J, Robinson K, Evans A, Smith D, Cunningham L, Millward R, Minnis H. The Strengths and Difficulties Questionnaire: a pilot study of a new computer version of the self-report scale. Eur Child and Adolesc Psychiatr 2003; 12(9): 14.

Von Klitzing K, Kelsay K, Emde RN, Robinson J, Schmitz S. Gender-specific characteristics of five-year-olds' play narratives and associations with behavior ratings. J Amer Acad Child Adolesc Psychiatr 2000; 39(8): 1017-23.

Warren S, Emde R, Sroufe A. Internal representations: predicting anxiety from children's play narratives. J Amer Acad Child and Adolescent Psychiatry 2000; 39(1): 100-7.

Warren S, Oppenheim D, Emde R. Can emotions and themes in children's play predict behavior problems? J Amer Acad Child Adolescent Psychiatr 1996; 35(10): 1331-7.

West P, Sweeting H, Der G, Barton J, Lucas C. Voice-DISC identified DSM-IV disorders among 15-year-olds in the West of Scotland. J Amer Academy Child Adolesc Psychiatr 2003; 42(8): 941-9.

Woolgar M, Steele H, Steele M, Yabsley S, Fonagy P. Children's play narrative responses to hypothetical dilemmas and their awareness of moral emotions. Brit J Dev Psychology 2001; 19(1): 115-28.

Correspondence: Dr Helen Minnis, University of Glasgow Section of Psychological Medicine, Caledonia House, Yorkhill NHS Trust, Glasgow G3 8SJ.

Telephone (+44) 01412019239

Email:h.minnis@clinmed.gla.ac.uk. 